\section{A closer look: secondary glaucoma more likely}

\section{To the editor:}

In the October issue of Lab Animal (Vol. 35, No.9), we published an article, "What's Your Diagnosis? Exophthalmos and corneal edema in a young ferret," in which we diagnosed an 8-week-old ferret with glaucoma in its right eye. Subsequently, Dr. Richard Smith, MD, D. Med. Sci., an ocular pathologist of mutant mouse eyes at The Jackson Laboratory (Bar Harbor, ME), contacted us with a different histopathological interpretation of the changes we described in the ferret's eye. In short, Dr. Smith believed the ocular changes leading to glaucoma were secondary, not primary. To clarify the matter, we sent the original histopathology slides to Dr. Smith for review. After discussing the slides with him, we feel it appropriate to revise our diagnosis.

Although focal edema of the cornea does occur, it is unusual. The right eye shown in Figure 1 (which was also Fig. 1 in the original article) is enlarged but neither edematous or exopthalmic. What we originally proposed to be corneal edema is actually corneal neovascularization, scarring, and thickening. This revised interpretation means that the corneal opacity in the right eye of the ferret in Figure 1 (incorrectly labeled in the original as the left eye) is a corneal scar, probably from a central corneal ulcer that the ferret had in the past. The original histological slides are sections through the peripheral cornea and show lesions from the edge of a

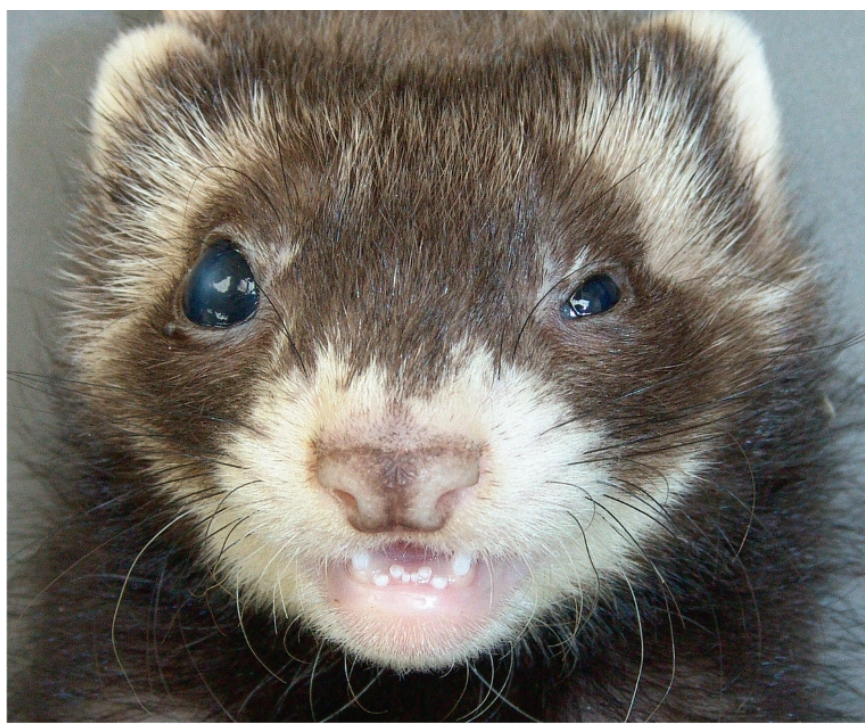

FIGURE 1 | An 8-week-old male ferret with an enlarged right eye that appears swollen. The condition, however, should not be called exophthalmos as this occurs when an eye of normal size is pushed forward by enlargement of the orbital contents. In this animal, the enlargement is due to secondary glaucoma.

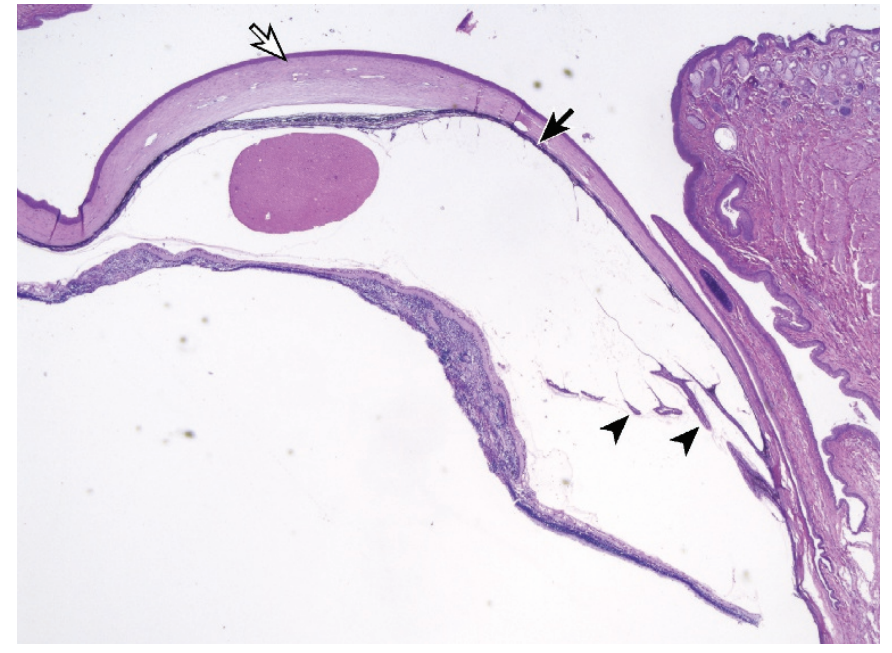

FIGURE 2 | Low-power photomicrograph of the whole anterior segment of the right eye in Figure 1. The white arrow indicates the cornea at three times the normal thickness with numerous (abnormal) corneal blood vessels. Peripherally the iris is attached to the cornea resulting in broad anterior synechiae (black arrow). Black arrowheads point to retracted ciliary processes that caused closure of ocular drainage channels and secondary glaucoma.

healed corneal injury. Serial sections through the eye would mostly likely show a corneal perforation site that had healed.

The corneal ulcer likely led to a prolonged period of inflammation resulting in vitreous traction that pulled on the ciliary processes (Fig. 2) and retina, detaching the retina. The invasion of new vessels into the vitreous is further evidence to support retinal detachment. The presence of numerous cells in the vitreous is highly abnormal

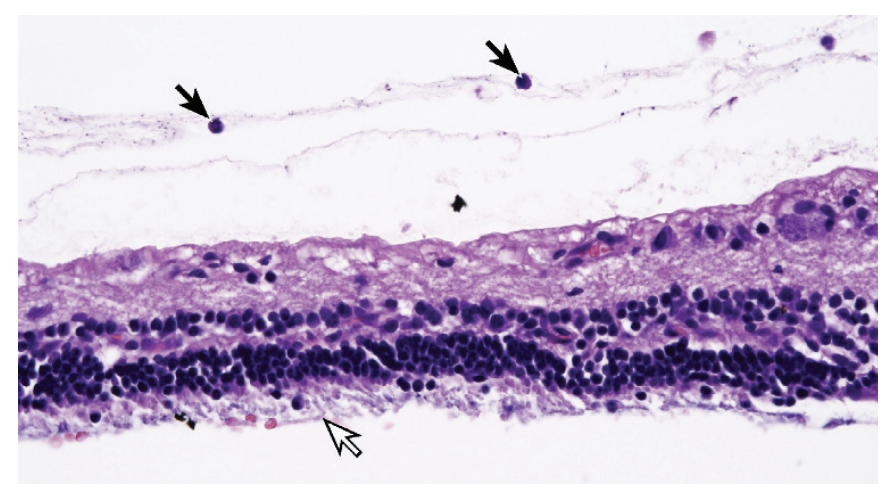

FIGURE 3 | High-power photomicrograph of the peripheral retina of the right eye in Figure 1. The peripheral retina is severely thinned. The white arrow indicates residual inner segments of the photoreceptors (probably about two-thirds of which are gone) and the absence of outer segments of photoreceptors. The two black arrows mark two of the many old macrophages/degenerating neutrophils present in the vitreous. 
and they are most likely old macrophages (Fig. 3). Sections of the central retina show severe distortion, which also suggests contraction and fibrosis of the vitreous. Moreover, the absence of outer segments of photoreceptors and the dearth of inner segments of photoreceptors (Fig. 3) suggest that the retina has been detached for many weeks or months. Considering the severe anterior synechiae (Fig. 2), it is likely this ferret had secondary glaucoma caused by synechial-induced closure of the ocular drainage channels.

In young mice, old healed corneal ulcers are common. These usually happen shortly after the eyes open (P14 in mice), a time when most rodents do not blink much. Consequently, blepharospasm, seen in adults with corneal ulcers, is not noticeable in very young mice. Because pre-weaned animals spend most of their time sleeping or suckling, animal caretakers may not notice corneal clouding, another indication of corneal ulcer in adult animals. Bedding, fecal pellets, and accidental littermate trauma are all causes of corneal ulcer in young mice, and are a likely source of the original corneal injury in this young ferret.
Kelli Boyd, DVM, PhD ${ }^{1}$, DAVCP, Richard S. Smith, MD, D. Med. Sci. ${ }^{2}$, Amy J. Funk, DVM ${ }^{1}$, Tiffani D. Rogers, DVM, DACLAM ${ }^{1}$ \& Rose Marie Dobbins ${ }^{1}$

${ }^{1}$ Department of Animal Resources, St. Jude Children's Research Hospital, Memphis, TN 38105. ${ }^{2}$ The Jackson Laboratory, Bar Harbor, ME 04609.

email:Amy.Funk@stjude.org

\section{Column Editor replies:}

In some tissues, anatomical details are relatively simple and easily analyzed histologically. The eye, however, is a complex structure with important regional anatomical variations that make histological interpretation challenging at best. If the orientation of the eye during sectioning is not carefully controlled, it is easy to make erroneous interpretations. For this and other reasons, eye pathology is a recognized subspecialty of pathology.

\section{Thomas M. Donnelly, DVM}

email:tom@tomvet.com 